\title{
Coulisses
}

Revue de théâtre

\section{Les derniers avatars musicaux du mythe de Faust}

\section{Emmanuel Reibel}

\section{CpenEdition}

Journals

Édition électronique

URL : https://journals.openedition.org/coulisses/273

DOI : $10.4000 /$ coulisses. 273

ISSN : 2546-9460

\section{Éditeur}

Presses universitaires de Franche-Comté

\section{Édition imprimée}

Date de publication : 31 décembre 2011

Pagination : 73-77

ISBN : 978-2-84867-404-9

ISSN : 1150-594X

Référence électronique

Emmanuel Reibel, «Les derniers avatars musicaux du mythe de Faust », Coulisses [En ligne], 43 | Automne 2011, mis en ligne le 30 novembre 2016, consulté le 29 décembre 2022. URL : http:// journals.openedition.org/coulisses/273; DOI : https://doi.org/10.4000/coulisses.273 


\title{
Les derniers avatars musicaux du mythe de Faust
}

\author{
Emmanuel Reibel
}

\section{NOTE DE L'ÉDITEUR}

Ce texte est une reprise du programme : Philippe Fénelon : Faust (2006), Théâtre du Capitole.

1 Né dans l'Allemagne luthérienne, le mythe de Faust connut une extraordinaire extension musicale au XIX siècle: en métamorphosant l'obscur alchimiste de la Renaissance en héros titanesque du désir et de la connaissance, la tragédie de Goethe hanta une génération d'artistes qui se révéla et se comprit à travers la figure faustienne. Tandis que Berlioz, Schumann ou Liszt, pour ne citer que les plus flamboyants, s'en nourrirent une vie durant, Gounod signa un opéra qui popularisa la tragédie goethéenne sur tous les continents. Mais il n'y avait aucune évidence à ce que le $\mathrm{xx}^{\mathrm{e}}$ siècle s'intéressât encore à Faust. Le sujet n'était-il pas éculé ? Opéra le plus joué au monde, parodié à travers de nombreuses opérettes, le Faust de Gounod était devenu bon gré mal gré l'archétype du genre lyrique, et le fameux « Air des bijoux » alimenta le répertoire de plus d'une Castafiore... Après la Symphonie des mille, par ailleurs, il semblait difficile de renchérir sur le degré d'extension et d'abstraction auquel Mahler avait hissé le mythe. Plus fondamentalement, l'optimisme et le dynamisme faustiens semblaient a priori peu en phase avec un siècle qui devait s'annoncer désenchanté, et qui mit en crise l'idée même de progrès à laquelle la figure de Faust avait fini par s'identifier.

2 Le Faust de Goethe fut bel et bien mis à distance au $\mathrm{xx}^{\mathrm{e}}$ siècle : mais contre toute attente, le mythe ne finit point d'enflammer l'imagination des musiciens. Après le Doktor Faust de Busoni, dans l'entre-deux-guerres, la deuxième moitié du siècle vit de nombreuses résurgences du sujet : s'y confrontèrent notamment Henri Pousseur (Votre Faust), Wolfgang Rihm (Doktor Faustus), Georges Aperghis (Faust et Randga), York Höller (Le Maître et Marguerite) ou Giacomo Manzoni (Doktor Faustus); depuis une quinzaine 
d'années, Faust est même l'une des figures mythiques les plus prisées des musiciens : suscitant le somptueux testament musical d'Alfred Schnittke (Historia von D. Johann Fausten, Hambourg, 1995), le récent opéra de John Adams outre-Atlantique (Doktor Atomic, San Francisco, 2005), un bel opéra de Pascal Dusapin, à la sombre diaprure métaphysique (Faustus, the last night, Berlin, 2006), une œuvre plus carnavalesque de Leonardo Balada (Faust-Bal, Madrid, 2009) ; et enfin ce Faust de Philippe Fénelon, créé au Théâtre du Capitole (Toulouse, 2007), à l'instigation de Nicolas Joël.

\section{L'ombre de Thomas Mann}

3 L'une des explications de cette embellie tient au renouvellement littéraire et philosophique du mythe. Après Oswald Spengler, qui vit en Faust l'archétype de l'Occident moderne dans sa propension à viser l'infini (tant dans l'ordre de la connaissance que dans celui du sentiment ou de la représentation du temps), au cœur du siècle, le magistral roman de Thomas Mann, Doktor Faustus, fit date en posant une question fondamentale : peut-on encore défendre l'Occident après la Shoah? Sur le plan politique, l'aspiration à l'Ordre apparue dans l'entre-deux-guerres a certes dégénéré en "pacte avec le diable». Mais la réflexion de Thomas Mann touche également l'art et la musique, dans la mesure où Faust, alias Adrian Leverkühn, n'est plus un alchimiste nécromancien mais un compositeur de musique moderne derrière lequel plane l'ombre de Schönberg. Artiste visionnaire imaginant des problèmes musicaux qu'il résout comme des parties d'échecs, Leverkühn est l'emblème d'une avant-garde radicale: il recourt à la loi implacable du nombre au point de clore effroyablement ses œuvres sur elles-mêmes. La musique moderne est le fruit d'un pacte avec le diable dont l'enjeu serait le génie mais le prix l'isolement.

4 Comment, en tant que musicien, ne pas être fasciné par l'imagination flamboyante de ce compositeur fictif? Ce n'est pas un hasard si toute la génération d'après-guerre, de Hanns Eisler à Alfred Schnittke, se nourrit de ce Doktor Faustus. Konrad Boehmer imagina de donner corps à l'une des «œuvres» dantesques d'Adrian Leverkühn, Apocalipsis cum figuris. Une bande magnétique à huit pistes, quatre percussionnistes, deux pianistes et trois chanteurs pop sont requis pour cette Apocalypse musicale (1984). Deux ans auparavant, le compositeur norvégien Arne Nordheim avait composé à la demande de Rostropovitch un Concerto pour violoncelle et orchestre sous-titré "Tenebrae » : la dernière section en fut inspirée par la description que Thomas Mann fait de la cantate composée par Leverkühn. L'Allemand Henze écrivit de son côté en 1997 son Troisième Concerto pour violon, sous-titré « Trois portraits d'après le roman Dr. Faustus de Thomas Mann ». À l'instar de la Faust-Symphonie de Liszt, qui présentait successivement les trois personnages principaux de la tragédie goethéenne, ce concerto évoque tour à tour "Esmeralda ", la femme par qui le héros-compositeur contracte la syphilis, « Das Kind Echo », le dernier amour de Leverkühn, et « Rudi S. », le violoniste à l'orchestre de Munich pour lequel Leverkühn avait composé un concerto de violon. Quant à l'Italien Giacomo Manzoni, il imagina, d'après le monumental Docteur Faustus de Thomas Mann, un opéra qui fut créé à la Scala de Milan en 1989. 


\section{Par-delà l'idéalisme goethéen}

5 Sur le modèle de Thomas Mann, puisant aux sources luthériennes du mythe de quoi miner l'idéalisme goethéen, les musiciens continuèrent à s'appuyer paradoxalement sur des sources anciennes pour mieux actualiser le sujet. Tandis que Hanns Eisler, à travers son livret d'opéra Johann Faustus, plaçait son héros dans le contexte de la « guerre des paysans » de la Renaissance germanique, pour asseoir une lecture marxiste du mythe (sans réussir à le composer en raison d'interdictions politiques), Alfred Schnittke travailla directement sur le Volksbuch de 1587 (le premier « livre populaire » à mettre en forme les légendes faustiennes) pour une cantate ensuite étendue aux dimensions d'un opéra. Dans cette Historia von D. Johann Fausten, qualifiée par son auteur de « Passion négative », Faust redevient l'Antéchrist de la Renaissance, l'assoiffé de toute-puissance qui réveille le spectre des totalitarismes récents. En revisitant pour sa part la tragédie de Marlowe, Pascal Dusapin explora l'aspect le plus sombre du mythe: mégalomane forcené et paranoïaque, totalement envoûté par l'ultime connaissance, son Faust est isolé dans une ultime nuit pour ne cesser d'incarner, avec l'ambition de totale domination, la démence narcissique - le trait saillant de notre culture selon le compositeur.

6 Un an auparavant, l'Américain John Adams avait été sollicité par l'Opéra de San Francisco pour « actualiser » le sujet. Le parcours du savant et de l'alchimiste était cette fois-ci inspiré par la vie du scientifique Robert Oppenheimer, père de la première bombe lancée en 1945 sur Hiroshima. Et voilà comment Faust, alias Doktor Atomic, devint, outre le démon de la toute-puissance technique, l'archétype de la science sans conscience aux conséquences les plus terrifiantes. Le succès contemporain de la figure faustienne, à nouveau liée à un opprobre théologique, moral ou métaphysique, s'explique donc par sa capacité à incarner la crise de la modernité occidentale. Ce n'est pas un hasard si, se penchant sur l'un des plus grands Faust du XIX siècle, celui de Lenau, Philippe Fénelon choisit de visiter un texte d'un profond pessimisme, le plus anti-goethéen qui soit. Son livret rend saillantes les aspirations insensées de Faust, c'est-à-dire le risque d'aliénation que court l'humanité dans toute entreprise de transgression des limites de la nature. Cyclique, l'opéra ne fait relancer dans l'épilogue les interrogations existentielles posées dans le prologue: le personnage de Görg devient un nouveau Faust qui grimpe à nouveau la montagne escarpée de ses doutes. Aucune réponse véritable n'est apportée aux inquiétudes du personnage principal, comme aux menaces qu'il représente, pour lui-même ou pour les autres.

\section{Quelle musique pour Faust?}

7 Par-delà ses enjeux philosophiques, le mythe présente une dimension spécifiquement musicale. Non seulement parce que la figure de Faust est depuis le cœur du $\mathrm{xx}^{\mathrm{e}}$ siècle souvent liée à celle du Leverkühn de Thomas Mann, et donc à celle de Schönberg comment se positionner par rapport au visage de la modernité qu'il incarne ? - mais parce que depuis plus longtemps encore, le diable est musicien. Chanteur ou violoniste à ses heures, il ne cesse de mener le bal, entre sérénades paillardes et danses sataniques. Mais quelle est la musique du diable? Si les romantiques jouèrent de la stridente quarte augmentée - qu'ils identifiaient au diabolus in musica-, chez le compositeur fictif de Thomas Mann, à rebours, «la dissonance exprime tout ce qui est 
élevé, grave, pieux, spirituel, alors que l'harmonie tonale est réservée au monde de l'enfer qui sous ce rapport représente le monde de la banalité et du lieu commun ». Dans Le Maitre et Marguerite de York Höller, opéra post-sériel créé à Paris en 1989, les parties «diaboliques» sont dominées par l'électroacoustique; dans l'Historia von D. Johann Fausten de Schnittke, au langage souvent néo-tonal, la marche au supplice de Faust se fait sur un tango méphistophélique chanté par une vulgaire diablesse de cabaret... L'ambiguïté tient à ce que les musiques de Méphistophélès sont souvent les plus audacieuses et les plus fascinantes. Ce qui renvoie, par-delà les différences stylistiques, à la question de l'inquiétant pouvoir de la musique.

8 Cette musique qui enivre les sens, est-elle capable d'enivrer la Mort? Tel est, depuis l'Orfeo de Monteverdi, l'interrogation qui sous-tend le genre de l'opéra. Or, puisqu'il est devenu un archétype de la tradition lyrique, Faust pose à tout compositeur d'aujourd'hui, bon gré mal gré, ce défi de l'opéra: le rapport à une mémoire quasi saturée, et la capacité du chant à conjurer la mort. Dès 1969, dans Votre Faust, Henri Pousseur affrontait la dimension méta-théâtrale du sujet. Dans cette œuvre expérimentale, un jeune compositeur rencontre un directeur de théâtre qui lui fait la diabolique commande d'un opéra. Il aura autant de temps et d'argent qu'il souhaitera, la seule condition étant d'écrire un Faust... Cette œuvre pose la question de la possibilité même de l'opéra - et du sujet faustien qui plus est - après les désenchantements idéologiques et artistiques $\mathrm{du}$ cœur $\mathrm{du} \mathrm{xx}^{\mathrm{e}}$ siècle. Latent à travers tous les Faust postérieurs, ce questionnement ressurgit dans le Faust de Philippe Fénelon: à travers la scénographie fantasmagorique de Pet Halmen, lorsque la bibliothèque du rôle-titre n'est composée que de volumes portant le nom de Faust; à travers la musique elle-même, lorsque les citations issues de la mémoire sonore saturent le discours. Proposer un Faust au début du xxie siècle relève bien du défi. Défi par rapport à une lourde tradition musicale, à digérer et sublimer; défi par rapport à la possibilité de la musique de se faire Sens, par-delà les éphémères sensations : si, comme chez Lenau, la nature se dérobe, si la science est vaine, la religion muette et la sensualité éphémère, la musique n'est-elle que cet instrument de fascination (méphistophélique) ou peut-elle incarner, elle, ce lieu de vérité (faustien) représentant la liberté créatrice? En voulant "échapper à la nuit de l'esprit ", l'opéra de Fénelon relève ce double défi en conjurant tous les fantasmes mortifères.

\section{INDEX}

oeuvrecitee Faust

Mots-clés : opéra, livret, avatar

Keywords : opera, libretto, avatar

Palabras claves : opera, libreto, avatar

Palavras-chave : opera, libreto, avatar 
AUTEURS

EMMANUEL REIBEL

Université de Paris Ouest-Nanterre-La Défense

Maître de conférences HDR en littérature comparée 